\title{
Effects of Online Sensory Feedback Restriction during the Training on Immediate and Long-Term Learning on a Finger Opposition Tapping Task
}

\author{
Maria Elisa Pimentel Piemonte ${ }^{1,2}$, Marcos Cammarosano Kopczynski ${ }^{1}$, Mariana Callil Voos ${ }^{2}$, Camila Souza \\ Miranda $^{2}$ Tatiana de Paula Oliveira ${ }^{2}$ \\ ${ }^{1}$ Department of Neuroscience and Behavior, Psychology Institute, University of São Paulo, Brazil \\ ${ }^{2}$ Department of Physiotherapy, Communication Science \& Disorders, Occupational Therapy, School of Medicine, \\ University of São Paulo, Brazil
}

Correspondence: Maria Elisa Pimentel Piemonte, Department of Neuroscience and Behavior, Psychology Institute, University of São Paulo, Brazil

\author{
Received: February 6, 2015 Accepted: February 26, 2015 Online Published: March 26, 2015 \\ doi:10.11114/jets.v3i3.665 \\ URL: http://dx.doi.org/10.11114/jets.v3i3.665
}

\begin{abstract}
Background: Online sensory feedback has been considered fundamental for motor learning. The sensory inputs experienced in previous attempts can be processed and compared to allow the online refinement of subsequent attempts, resulting on performance improvement. However, numerous studies have provided direct and indirect evidence that learning new motor abilities is possible, regardless of online sensory feedback. Objective: The purpose of this study was to investigate the influence of online sensory feedback on learning a new motor skill. Methods: We investigated the immediate and the 28-day delayed performance of a single-session training (4 blocks of 600 movements) performed under 4 different conditions of online sensory restriction: (1) no restriction; (2) visual input restriction; (3) visual and tactile input restriction and (4) total input restriction (mental practice), in 40 young healthy participants (mean age 26.6 \pm 3.6 years). The task consisted of performing finger opposition sequences using the dominant hand (right). Results: The training condition did not influence immediate or delayed performance in terms of accuracy or speed. Conclusion: As all conditions resulted in a similar amount of performance improvement, the level of online feedback available during training did not affect the learning process. Probably, according to the sensory inputs availability on the training conditions, equally efficient strategies are developed to improve performance in terms of speed and accuracy.
\end{abstract}

Keywords: motor learning, mental practice, internal model, sequence movement, motor skill, sensory disruption

\section{Introduction}

Motor learning is considered the main modality of the implicit system, involving acquisition by physical practice without direct access to conscious recollection as facts or data (Squire \& Zola, 1996). It develops with practice over a period, with repetition (Vidoni \& Boyd, 2007). These characteristics explain the need for extensive training in order to attain satisfactory performance in any motor task, such as practicing sports or playing musical instruments (Kantak \& Winstein, 2012).

Repetition allows the online refinement of the next attempts by considering the sensory feedback from previous attempts.The desired movements are compared with the performed movements and after several attempts and corrections, a more efficient performance is finally achieved (Shabbott \& Sainburg, 2010). Then, the performance becomes guided by anticipatory control based on a forward internal model for sensorimotor prediction (Tin \& Poon, 2005). This is possible because the offline sensory experience is built, considering the knowledge of previous attempts (Miall \& Wolpert, 1996). Therefore, most consequences of the movement can be predicted. At this point, practice no longer constitutes of merely repetition, but encompasses online sensory feedback as fundamental in motor learning, at least in the initial phases of the process. Thus, according to classical theories about motor learning, online sensory feedback is extremely important for reaching a higher level of performance (Pavlides, Miyashita, \& Asanuma, 1993; Schmidt \& Bjork, 1992).

Consequently, strategies to increase online sensory feedback are extensively used in motor training to improve learning, particularly during the initial phases of training (Keller, Lauber, Gehring, Leukel, \& Taube, 2014; Lauber \& Keller, 2014; Pataky et al., 2010; York, Perell-Gerson, Barr, Durham, \& Roper, 2009). Indeed, the results of several studies 
have confirmed that both visual and tactile feedback are fundamental in learning new skills (Blank, Heizer, \& von Voss, 1999; Land, 2006; Schween, Taube, Gollhofer, \& Leukel, 2014). Nevertheless, recent studies have called into question whether online feedback is, in fact, fundamental to the learning process (Wierinck, Puttemans, \& van Steenberghe, 2006; Wu et al., 2011). Studies showing that (1) patients with sensory impairments do not show difficulty learning new motor skills (Missaoui \& Thoumie, 2013; Riva et al., 2014); (2) untrained segments are able to improve their performance on a new motor skill after the training of a distinct body segment, therefore, without online sensory feedback (Harley \& Prilutsky, 2012), and (3) learning by mental practice (Gentili, Papaxanthis, \& Pozzo, 2006; Schuster et al., 2011), i.e. the repeated use of motor or kinesthetic imagery by internal rehearsal of movement from a first-person perspective without any overt physical movement and, consequently, with zero online sensory feedback indicate that the feedback function in motor learning is not yet fully understood. Besides, studies that compared the activation of brain areas during physical and mental practice showed parietal sensory areas to be less activated during mental imagery of simple movements (Lotze \& Halsband, 2006). These data confirm the absence of online sensory feedback on such training.

Given the importance of further knowledge on this issue, in order to improve training techniques, the present study focused on comparing the immediate and long-term results of training, performed under four sets of distinct online sensory feedback conditions, in a motor task learning. Training conditions were optimized for the comparison of a first control condition, with no online sensory restriction, to a second condition, with restricted online visual feedback, a third, with both online visual and tactile restrictions, and a last condition, based on mental practice, in which no online sensory feedback was allowed.

Thus, the differences in immediate or long-term performance, stemming from different online sensory training conditions, would indicate that the level of online feedback available during training does, in fact, affect the learning process. In contrast, if all conditions promote the same level of performance improvement, this would indicate that a new motor skill would be learned regardless of the level of online sensory feedback provided during the training, even in the initial phase of the process.

\section{Methods}

\subsection{Participants}

A sample size calculation based on a pilot study (Oliveira, Geressaite \& Piemonte, 2006) showed that 40 adults (10 in each group) would be sufficient to reach a power greater than $.9(\alpha=.05 ; E S=.91)$. A total of 40 healthy individuals took part including 20 men and 20 women, with mean age of $26.6 \pm 3.6$ years, right-hand dominance and mean schooling level of $15 \pm 2$ years. Individuals presenting sensory and/or motor and/or cognitive disturbances were excluded from the study, as were individuals having previous extensive practice of hand movements, such as musicians and typists.

As stipulated by the institutional review board for the protection of human participants at the affiliated university Ethics Committee for Research Project Analysis of the Hospital das Clínicas - São Paulo, Brazil, written informed consent was obtained from all participants.

\subsection{Task and Procedure}

\subsubsection{The Finger-to-Thumb Opposition Sequence (FOS) Task}

The motor task consisted of the finger-to-thumb opposition sequences previously described (Karni et al., 1998). Two sequences of equal length and complexity were used; each sequence was the reverse of the other. The fingers were numbered 1 to 4 , with 1 designating the index finger and 4 the little finger; the 4-1-3-2-4 sequence was used as the trained sequence (TS), and 4-2-3-1-4 was used as the untrained sequence (UTS). Participants were instructed to oppose the fingers to the thumb in the given 5-movement sequence "as accurately and quickly as possible". They performed the task while seated comfortably in front of the desk where they could support their elbows and forearms. Task performance in terms of speed and accuracy was recorded by a computer-monitored device attached to the finger tips. The number of correct sequences per minute was used as a measure of performance.

\subsubsection{Procedure}

Participants were assessed in six individual sessions that occurred on five different days. The first session consisted of training and assessments (day 1). The second (day 4), third (day 7), fourth (day 14), and fifth sessions (day 28) consisted of assessments only. Each assessment lasted for 1 minute and was started and stopped by an auditory signal. On each assessment, participants were instructed to tap the sequence continuously until they heard the stop signal. If any errors occurred, participants were instructed to continue the task without pausing. Both TS and UTS were performed with the right hand.

\subsubsection{Before-Training (BT) Assessment}


Initially, participants were asked to memorize the sequence of numbers of the TS and UTS, printed on a sheet. After 2 minutes, retention was verified by asking the participants to repeat the sequences verbally. After the participants had been able to memorize the sequences, they performed 10 trials of each finger-to-thumb opposition sequence. Next, the first motor performance assessment was conducted.

2.2.2.2 Training

Following the first assessment, participants were randomly split into 4 groups:

Control group (CT): Participants of this group carried out the training without any online sensory restriction;

Visual restriction group (VR): Participants of this second group performed the training blindfolded, with no online visual feedback;

Visual and tactile restriction group (VTR): Participants were blindfolded and wore a glove with special cotton lining to reduce the sensory feedback of the finger tips when opposing to each other;

Mental practice group (MP): Participants of this fourth group completed their mental practice using kinesthetic imagery of the same task, while keeping their eyes closed and fingers interlocked to prevent any attempt of physical practice. The instructions, that emphasized the kinesthetic imagery (i.e., imagining the participants's own action), preventing the use of visual imagery (internal rehearsal of movement from a third-person perspective, i.e., visualizing actions outside of the subject's own body), were "imagine you are moving your thumb against each of your fingertips in the same order as the numbers in the sequence, as fast as you can, without making mistakes".

The instructions for CT, VR and VTR were "move your thumb against each of your fingertips in the same order as the numbers of the sequence, as fast as you can, without making mistakes."

The training of the four groups consisted of 4 consecutive blocks (B1, B2, B3, and B4) of 120 repetitions of the TS (600 finger-to-thumb opposition movements per block). Rest intervals of 2 minutes were allowed between the blocks. On CT, VR and VTR groups, the number of sequences was controlled by a computer, while in the MP group, it was controlled by examiner, based on the participant's response, who verbalized the last item of the sequence aloud upon mental completion of each sequence.

\subsubsection{After-Training Performance Assessments}

The TS and UTS performances were recorded with an after-training assessment (AT), five minutes after the end of training, to examine immediate learning. In addition, assessments were completed at 4 (4 dAT), 7 (7 dAT), 14 (14dAT) and 28 (28 dAT) days after the end of training to examine long term learning. The same conditions were employed for all assessments.

\subsection{Statistical Analysis}

The demographic characteristics and performance in baseline assessment (BT) of the four groups (CT, VR, VTR and MP) were compared using one-way ANOVAs.

The Kolmogorov-Smirnov and Levene tests were used to assess the normality and homogeneity of variance, respectively, for all measures.

To analyze the training acquisition, i.e., performance improvement on TS during 4 training blocks, we used a 4 [Group] $\mathrm{X} 4$ [Block] mixed-design ANOVA with Group as the between-subject factor [CT, VR, VTR and MP] and Block [B1, B2, B3, B4] as the within-subject factor. The number of correct sequences per minute for each training block was used as the dependent variable.

To analyze learning, we used a 4[Group] X 2 [sequence] x 6 [Assessment Time Points] mixed-design ANOVA with Group [CT, VR, VTR and MP] as the between-subject factor and Sequence [TS and UTS] and Assessment Time Point [BT, AT, 4 dAT, $7 \mathrm{dAT}, 14 \mathrm{AT}$ and $28 \mathrm{dAT}$ ] as the within-subject factor. The number of correct sequences per minute was used as the dependent variable.

The effect sizes $(E S)$ were calculated for all ANOVAs (alpha $=.05$ ). A Tukey HSD post-hoc test was performed to account for multiple comparisons. The statistical software Statistica 10.0 from StatSoft (USA) was used for all analyses, and $p$-values below $5 \%$ were considered statistically significant.

\section{Results}

The analysis of participants demographic data and performance on baseline assessment (AT) revealed no statistically significant differences [ES =.90] between the groups [CT, VR, VTR and MP].

\subsection{Acquisition during the Training Session}

The analysis of performance during the training session in the four groups (Figure 1), revealed a block effect only $[F$ 
$(3,108)=151.59, p<.000001, E S=.99]$. Post-hoc comparisons using the Tukey HSD test indicated that number of correct sequences on the second $(\mathrm{p}=.00001)$, third blocks $(\mathrm{p}=.00001)$ and fourth blocks $(\mathrm{p}=.00001)$ were significantly higher than on the first block for all groups. As expected, there was an equivalent progressive increase on the FOS task performance during the training independently of the online sensory feedback restriction.
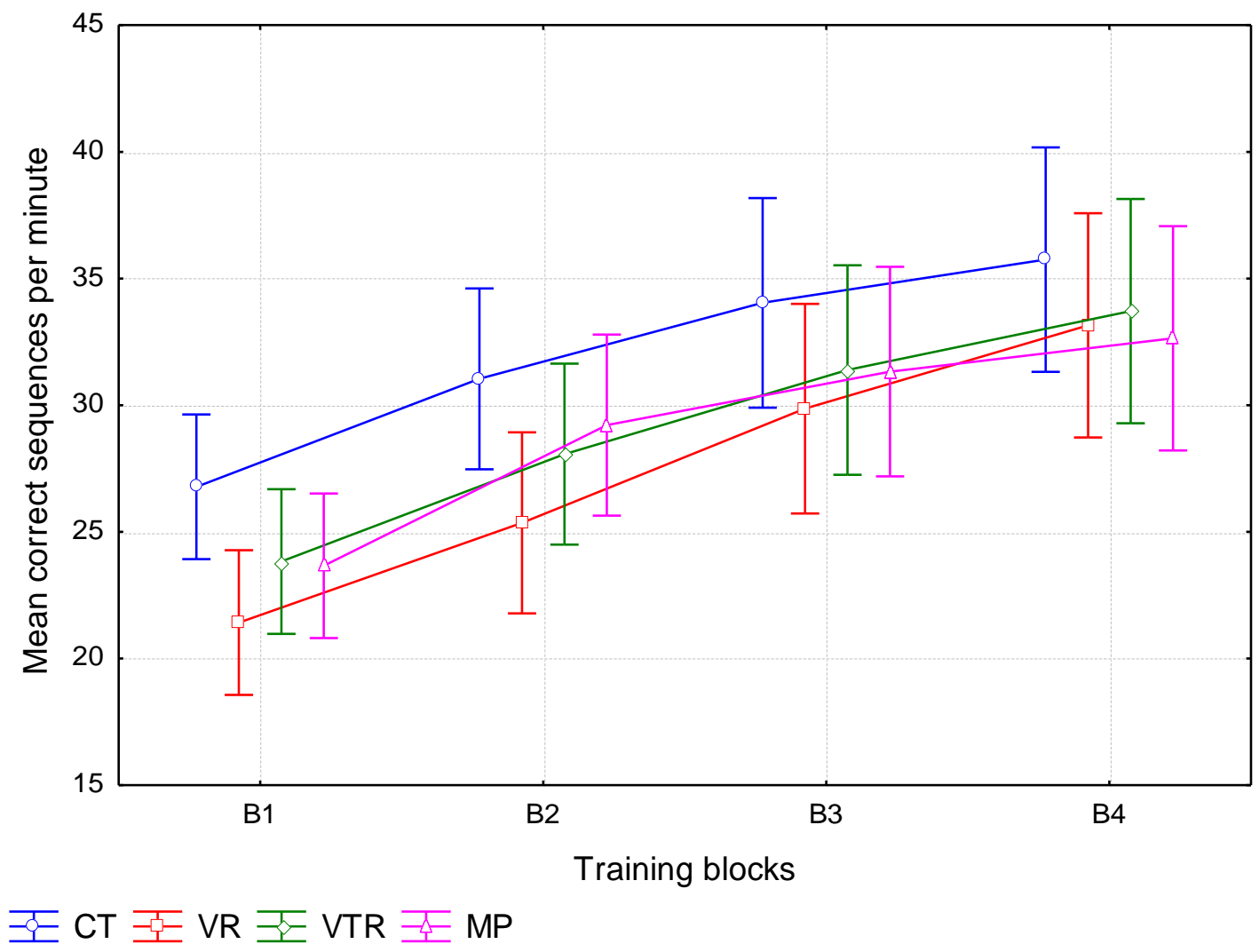

Figure 1. Mean scores and standard error bars for trained sequence (TS) performance in terms of correct sequences per minute during the training. Note. $\mathrm{B} 1=$ training block $1 ; \mathrm{B} 2=$ training block $2 ; \mathrm{B} 3=\operatorname{training}$ block 3 ; $\mathrm{B} 4=$ training block 4; $\mathrm{CT}=$ control group; $\mathrm{MP}=$ mental practice; $\mathrm{VR}=$ visual restriction; $\mathrm{VTR}=$ visual and tactile restriction.

\subsection{Learning after the Training Session}

Initially, all groups presented the same performance for both trained and untrained sequences, in terms of speed and accuracy.

The analysis of performance, in terms of number of correct sequences in six assessment time points (Figure 2), showed a significant interaction amongst group [CT, VR, VTR and MP], assessment [BT, AT, 4 dAT, 7 dAT, 14 AT and 28 dAT] and sequence [TS and UTS], $(F(15,18)=2.58, p<.001, E S=.90)$. Nonetheless, the Tukey HSD test revealed no significant differences between groups in each of the assessments for the TS.

The only aspects that were different between groups were related to the UTS performance. All groups, except MP, showed a significant difference between TS and UTS speed in AT assessment (CT: $p<.0001$; VR: $p<.01$ and VTR: $p$ $<.01)$, i.e, as expected, immediately after the end of training, the TS performance was higher compared to UTS. Regardless of this fact, all groups showed significant performance improvement on the UTS after the training, starting from 7dAT assessment (CT: $p<.00001$; VR: $p<.00001$; VTR: $p<.00001$ and MP: $p<.00001$ ), which remained in 14dAT assessment (CT: $p<.00001$; VR: $p<.00001$; VTR: $p<.00001$ and MP: $p<.00001$ ), and 28dAT assessment (CT: $p<.00001$; VR: $p<.00001$; VTR: $p<.00001$ and MP: $p<.00001)$. 


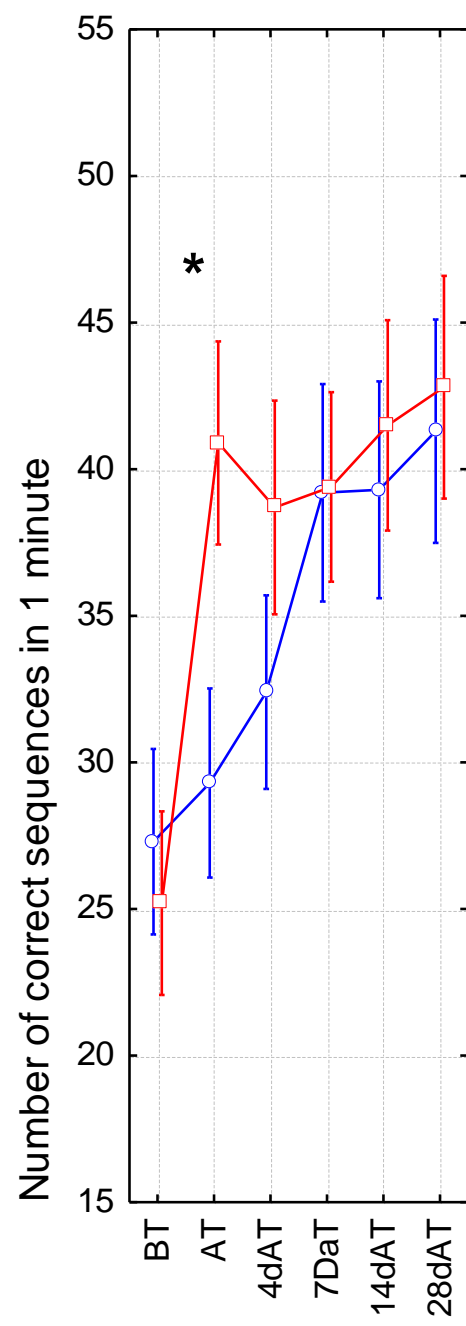

Assessments CT

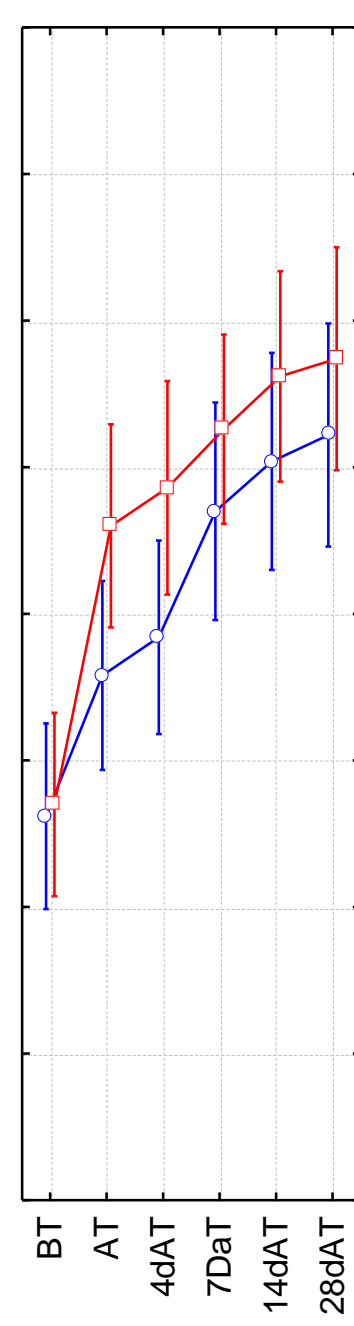

Assessments

MP

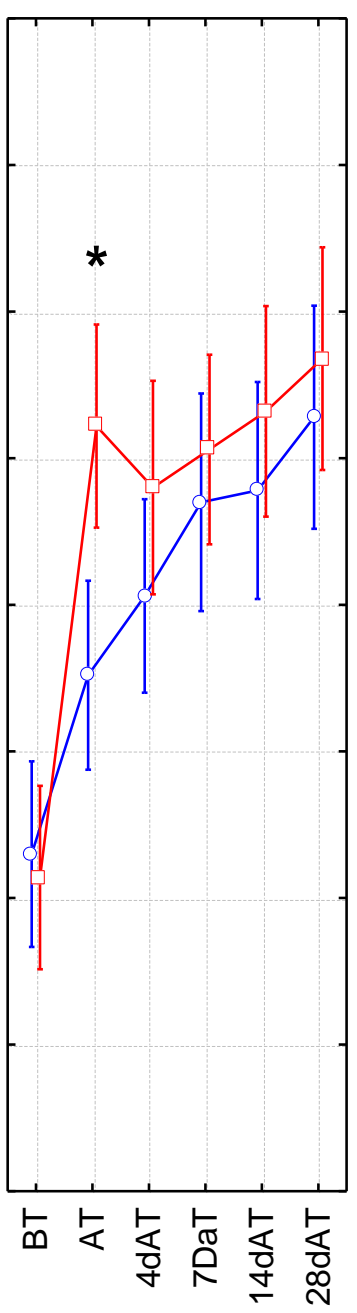

Assessments

VR

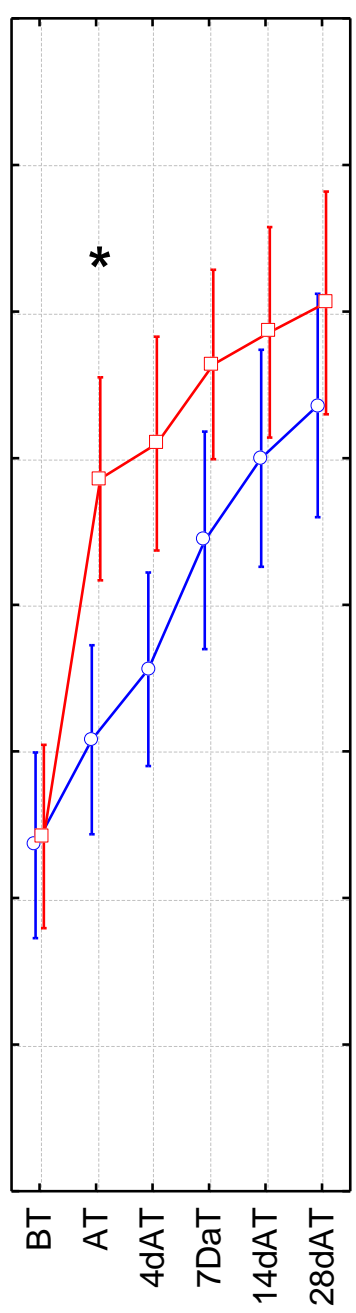

Assessments VTR

\section{코 UTS 코TS}

Figure 2. Mean scores and standard error bars for trained sequence (TS) and untrained sequence (UTS) performance in terms of correct sequences per minute Note. BF = before training; AT = immediately after training; $4 \mathrm{dAT}=4 \mathrm{days}$ after; $7 \mathrm{dAT}=7$ days after; $14 \mathrm{dAT}=14$ days after; $28 \mathrm{dAT}=28$ days after; $\mathrm{CT}=$ control group; $\mathrm{MP}=$ mental practice; VR = visual restriction; VTR $=$ visual and tactile restriction; ${ }^{*} p=.001$ (sequence effect, Tukey HSD post hoc test).

Thus, all training conditions promoted similar immediate and long-term improvements of performance, regardless of the level of online sensory feedback. Additionally, only mental practice promoted the similar level of improvement between TS and UTS immediately after the end of training.

\section{Discussion}

The present study investigated the effects of online sensory feedback on the immediate and long-term learning in healthy adults. We proposed an experimental design, with progressive restriction of online sensory feedback during the training. The most restrictive condition was mental practice, in which no movement was allowed and, consequently, no online sensory feedback. Thus, differences in immediate or long-term performance, stemming from distinct online sensory training conditions, would indicate that the level of online feedback available during the training did, in fact, affect the learning process.

Our results showed that all training conditions produced the same amount of learning. Independently of the level of 
online sensory feedback restriction, all groups obtained the same motor performance improvement on the trained movement sequence, immediately and on the retention assessment 28 days after the end of training. Performance during training was also the same for all groups. Therefore, the online sensory feedback restrictions imposed during the training had no limiting effect on learning.

This suggests that the performance on a new skill can be improved regardless of online sensory feedback, even in the initial phases of learning. For visual (VR) and visual-tactile restriction (VTR) conditions, this result could be attributed to the processing of other intact sensory inputs, such as proprioceptive information, which could compensate for the visual and tactile inputs absence (Norman \& Bartholomew, 2011; Ozdemir, Pourmoghaddam, \& Paloski, 2013; Wong, Gnanakumaran, \& Goldreich, 2011). In fact, studies showed that, in cases of absence or limitations in one or more categories of sensory input in healthy participants (Levy-Tzedek et al., 2012) or in patients with neurological diseases affecting the sensory system (Missaoui \& Thoumie, 2013; Riva et al., 2014), mechanisms based on the increase in processing of the remaining sensory inputs could be activated to compensate the deficiency to control the performance of well-learned tasks. The new evidence emerging from the present study suggests that similar compensatory mechanisms are also efficient during the leaning of a novel skill.

However, this hypothesis does not explain the results obtained after mental practice. In this condition, due to the absence of movement, there is no online sensory feedback. Thus, the use of compensatory mechanisms among distinct sensory categories is not a plausible hypothesis to explain the same level of performance improvement obtained after the training. In our opinion, the more plausible explanation for this fact consists of the efficient use of offline sensory information, i.e., the use of memorized sensory information obtained by previous similar experiences to guide the learning process (Rieger, 2012; Song, 2009). Thus, in the total absence of online sensory inputs, the compensatory mechanisms activate the search for offline sensory information that could be efficient to develop the enhancement of performance of a new motor skill. In this situation, a forward sensory model would be used to predict the sensory consequences of the movements (Avanzino et al., 2009; Desmurget et al., 2009; Grush, 2004; Haruno, Wolpert, \& Kawato, 2001; Tian \& Poeppel, 2012). Thus, action would be preplanned via an ideomotor-like mechanism, in which movements could be triggered and facilitated by the imaginary anticipation of their effects (Bernardi et al., 2013).

This hypothesis could also explain another interesting result from the present study: the mental practice was the unique training condition that, immediately after the training, promoted similar levels of improvement for the TS and UTS, which did not show statistically significant difference. For the other three groups (CT, VR and VTR), as expected, the TS speed on this assessment (AT assessment) was higher. Thus, the learning during mental practice, guided by offline sensory information and based on more inferential learning strategies, could be more flexible and easier to generalize (Miall \& Wolpert, 1996). This fact could explain the higher transfer of performance between TS and UTS. A previous study with children (Asa, Melo, \& Piemonte, 2014) confirmed that the transfer of performance between sequences and hands was higher with mental practice in comparison with physical practice. Considering its independence on online sensory feedback, mental practice could be an important alternative strategy to promote motor learning, in the cases of the sensory disruption. To clarify this hypothesis, new studies will be necessary to investigate the mental practice outcomes on acquisition of a new skill in patients with sensory dysfunction.

Our results, although consistent, are limited to behavioral measures, i.e., analysis of motor performance. New studies that, besides behavioral measures, analyse possible changes on brain activation on training conditions with distinct levels and categories of sensory information are fundamental to elucidate the role of the online and offline sensory information in motor learning and its compensatory mechanisms.

\section{Conclusion}

In conclusion, based on the results of the present study, the restriction of online sensory information during the training did not impair the learning of a new finger-to-thumb opposition sequences task in healthy adults.

\section{References}

Asa, S. K. P., Melo, M. C. S., \& Piemonte, M. E. (2014). Effects of Mental and Physical Practice on a Finger Opposition Task Among Children. Research Quarterly Exercise and Sport, 85(3), 308-315. http://dx.doi.org/10.1080/02701367.2014.931557

Avanzino, L., Giannini, A., Tacchino, A., Pelosin, E., Ruggeri, P., \& Bove, M. (2009). Motor Imagery Influences the Execution of Repetitive Finger Opposition Movements. Neuroscience Letters, 466(1), 11-15. http://dx.doi.org/10.1016/j.neulet.2009.09.036

Bernardi, N. F., De, M., Trimarchi, P. D., Chielli, A., \& Bricolo, E. (2013). Mental Practice Promotes Motor Anticipation: Evidence from Skilled Music Performance. Frontiers in Human Neuroscience, 7, 451. http://dx.doi.org/10.3389/fnhum.2013.00451 
Blank, R., Heizer, W., \& von Voss, H. (1999). Externally Guided Control of Static Grip Forces by Visual Feedback-Age and Task Effects in 3-6-Year Old Children and in Adults. Neuroscience Letters, $271(1)$, 41-44. http://dx.doi.org/10.1016/S0304-3940(99)00517-0

Desmurget, M., Reilly, K. T., Richard, N., Szathmari, A., Mottolese, C., \& Sirigu, A. (2009). Movement Intention after Parietal Cortex Stimulation in Humans. Science, 324(5928), 811-813. http://dx.doi.org/10.1126/science.1169896

Gentili, R., Papaxanthis, C., \& Pozzo, T. (2006). Improvement and Generalization of Arm Motor Performance through Motor Imagery Practice. Neuroscience, 137(3), 761-772. http://dx.doi.org/10.1016/j.neuroscience.2005.10.013

Grush, R. (2004). The Emulation Theory of Representation: Motor Control, Imagery, and Perception. Behavioral and Brain Sciences, 27(3), 377-396. http://dx.doi.org/10.1017/S0140525X04000093

Harley, L. R., \& Prilutsky, B. I. (2012). Transfer of Learning between the Arms during Bimanual Reaching. Conference proceedings: Annual Internacional Conference of the IEEE Engineering in Medicine and Biology Society, 6785-6788. http://dx.doi.org/10.1109/EMBC.2012.6347552

Haruno, M., Wolpert, D. M., \& Kawato, M. (2001). Mosaic Model for Sensorimotor Learning and Control. Neural Computation, 13(10), 2201-2220. http://dx.doi.org/10.1162/089976601750541778

Kantak, S. S., \& Winstein, C. J. (2012). Learning-Performance Distinction and Memory Processes for Motor Skills: a Focused Review and Perspective. Behavioural Brain Research, 228(1), $219-231$. http://dx.doi.org/10.1016/j.bbr.2011.11.028

Karni, A., Meyer, G., Rey-Hipolito, C., Jezzard, P., Adams, M. M., Turner, R., \& Ungerleider, L. G. (1998). The Acquisition of Skilled Motor Performance: Fast and Slow Experience-Driven Changes in Primary Motor Cortex. Proceedings of the National Academy of Science of the United States of America, 95(3), 861-868. http://dx.doi.org/10.1073/pnas.95.3.861

Keller, M., Lauber, B., Gehring, D., Leukel, C., \& Taube, W. (2014). Jump Performance and Augmented Feedback: Immediate benefits and Long-Term Training Effects. Human Movement Science, 36, 177-189. http://dx.doi.org/10.1016/j.humov.2014.04.007

Land, M. F. (2006). Eye Movements and The Control of Actions in Everyday Life. Progress in Retinal and Eye Research, 25(3), 296-324. http://dx.doi.org/10.1016/j.preteyeres.2006.01.002

Lauber, B., \& Keller, M. (2014). Improving Motor Performance: Selected Aspects of Augmented Feedback in Exercise and Health. European Journal of Sport Science, 14(1), 36-43. http://dx.doi.org/10.1080/17461391.2012.725104

Levy-Tzedek, S., Novick, I., Arbel, R., Abboud, S., Maidenbaum, S., Vaadia, E., \& Amedi, A. (2012). Cross-sensory Transfer of Sensory-Motor Information: Visuomotor Learning Affects Performance on an Audiomotor Task, Using Sensory-Substitution. Scientific Reports, 2, 949. http://dx.doi.org/10.1038/srep00949

Lotze, M., \& Halsband, U. (2006). Motor Imagery. Journal of Physiologgy-Paris, 99(4-6), 386-395. http://dx.doi.org/10.1016/j.jphysparis.2006.03.012

Miall, R. C \& Wolpert, D. M. (1996). Forward Models for Physiological Motor Control. Neural Networks, 9(8), 1265-1279. http://dx.doi.org/10.1016/S0893-6080(96)00035-4

Missaoui, B., \& Thoumie, P. (2013). Balance Training in Ataxic Neuropathies. Effects on Balance and Gait Parameters. Gait \& Posture, 38(3), 471-476. http://dx.doi.org/10.1016/j.gaitpost.2013.01.017

Norman. J. F., \& Bartholomew, A. N. (2011). Blindness Enhances Tactile Acuity and Haptic 3-D Shape Discrimination. Attention, Perception, \& Psychophysics, 73(7), 2323-2331. http://dx.doi.org/10.3758/s13414-011-0160-4

Oliveira, A., Geressait, L., \& Piemonte, M. E. P. (2006). Is Feedback Really Fundamental to Learning of a New Motor Skill? in: Proceedings of the 5th Forum of European Neuroscience (pp.62-62). Federation of European Neuroscience Societies. Viena.

Ozdemir, R. A., Pourmoghaddam, A., \& Paloski, W. H. (2013). Sensorimotor Posture Control in the Blind: Superior Ankle Proprioceptive Acuity Does Not Compensate for Vision Loss. Gait \& Posture, 38(4), 603-608. http://dx.doi.org/10.1016/j.gaitpost.2013.02.003

Pataky, Z., de León Rodriguez, D., Allet, L., Golay, A., Assal, M., Assal, J. P., \& Hauert, C. A. (2010). Biofeedback for Foot Offloading in Diabetic Patients with Peripheral Neuropathy. Diabetic Medicine, 27(1), 61-64. http://dx.doi.org/10.1111/j.1464-5491.2009.02875.x

Pavlides, C., Miyashita, E., \& Asanuma, H. (1993). Projection from the Sensory to the Motor Cortex is Important in Learning Motor Skills in the Monkey. Journal of Neurophysiology, 70(2), 733-741. 
Rieger, M. (2012). Motor Imagery in Typing: Effects of Typing Style and Action Familiarity. Psychonomic Bulletin \& Review, 19(1), 101-107. http://dx.doi.org/10.3758/s13423-011-0178-6

Riva, N., Faccendini, S., Lopez, I. D., Fratelli, A., Velardo, D., Quattrini, A., \& Fazio, R. (2014). Balance Exercise in Patients with Chronic Sensory Ataxic Neuropathy: a Pilot Study. Journal of Peripheral Nervous System, 19(2), 145-151. http://dx.doi.org/10.1111/jns5.12065

Schmidt, R. A., \& Bjork, R. A. (1992). New Conceptualizations of Practice: Common Principles in Three Paradigms Suggest New Concepts for Training. Psychological Science, 3(4), 207-217. http://dx.doi.org/10.1111/j.1467-9280.1992.tb00029.x

Schuster, C., Hilfiker, R., Amft, O., Scheidhauer, A., Andrews, B., Butler, J., \& Ettlin, T. (2011). Best Practice for Motor Imagery: A Systematic Literature Review on Motor Imagery Training Elements in Five Different Disciplines. BMC medicine, 9, 75. http://dx.doi.org/10.1186/1741-7015-9-75

Schween, R., Taube, W., Gollhofer, A., \& Leukel, C. (2014). Online and Post-Trial Feedback Differentially Affect Implicit Adaptation to A Visuomotor Rotation. Experimental Brain Research, 232(9), 3007-3013. http://dx.doi.org/10.1007/s00221-014-3992-z

Shabbott, B. A., \& Sainburg, R. L. (2010). Learning a Visuomotor Rotation: Simultaneous Visual and Proprioceptive Information is Crucial for Visuomotor Remapping. Experimental Brain Research, 203(1), 75-87. http://dx.doi.org/10.1007/s00221-010-2209-3

Song, S. (2009). Consciousness and the Consolidation of Motor Learning. Behavioural Brain Research, 196(2), 180-186. http://dx.doi.org/10.1016/j.bbr.2008.09.034

Squire, L. R., \& Zola, S. M. (1996). Structure and Function of Declarative and Nondeclarative Memory Systems. Proceedings of the National Academy Sciences of the United States of America, 93, 13515-13522. http://dx.doi.org/10.1073/pnas.93.24.13515

Tian, X., \& Poeppel, D. (2012). Mental Imagery of Speech: Linking Motor and Perceptual Systems through Internal Simulation and Estimation. Frontiers in Human Neuroscience, 6, 314. http://dx.doi.org/10.3389/fnhum.2012.00314

Tin, C., \& Poon, C. S. (2005). Internal Models in Sensorimotor Integration: Perspectives from Adaptive Control Theory. Journal of Neural Engineering, 2(3), S147-163. http://dx.doi.org/10.1088/1741-2560/2/3/S01

Vidoni, E. D., \& Boyd, L. A. (2007). Achieving Enlightenment: What Do We Know about The Implicit Learning System and Its Interaction with Explicit Knowledge? Journal of Neurologic Physical Therapy, 31(3), 145-154. http://dx.doi.org/10.1097/NPT.0b013e31814b148e

Wierinck, E., Puttemans, V., \& van Steenberghe, D. (2006). Effect of Reducing Frequency of Augmented Feedback on Manual Dexterity Training and Its Retention. Journal of Dentistry, 34(9), 641-647. http://dx.doi.org/10.1016/j.jdent.2005.12.005

Wong, M., Gnanakumaran,V., \& Goldreich, D. (2011).Tactile Spatial Acuity Enhancement in Blindness: Evidence for Experience-Dependent Mechanisms. The Journal of Neuroscience, 31(19), 7028-7037. http://dx.doi.org/10.1523/JNEUROSCI.6461-10.2011

Wu, W. F. W., Young, D. E., Schandler, S. L., Meir, G., Judy, R. L. M., Perez, J., \& Cohen, M. J. (2011). Contextual Interference and Augmented Feedback: Is There an Additive Effect for Motor Learning? Human Movement Science, 30(6), 1092-1101. http://dx.doi.org/10.1016/j.humov.2011.02.004

York, R. M., Perell-Gerson, K. L., Barr, M., Durham, J., \& Roper, J. M. (2009). Motor Learning of a Gait Pattern to Reduce Forefoot Plantar Pressures in Individuals with Diabetic Peripheralneuropathy. PM \& R: the Journal of Injury, Function, and Rehabilitation, 1(5), 434-441. http://dx.doi.org/10.1016/j.pmrj.2009.03.001

This work is licensed under a Creative Commons Attribution 3.0 License. 